\title{
Imunodeficiência comum variável associada à doença de Crohn-like: relato de caso e revisão da literatura
}

\author{
Common variable immunodeficiency associated with Crohn-like disease: \\ case report and literature review
}

\author{
Larissa Cristina Tavares de Castro $(\mathbb{D} 1 \bowtie$, Zamir Calamita $(\mathbb{D}) 1$
}

1 Faculdade de Medicina de Marília (FAMEMA). Marília, São Paulo, Brasil

Como citar este artigo (How to cite this article):

de Castro LCT, Calamita Z. Imunodeficiência comum variável associada à doença de Crohn-like: relato de caso e revisão da literatura (Common variable immunodeficiency associated with Crohn-like disease: case report and literature review). Sci Med. 2019;29(1):e32159. https:// doi.org/10.15448/1980-6108.2019.1.32159

\section{RESUMO}

OBJETIVOS: relatar o caso de um paciente diagnosticado com imunodeficiência comum variável e doença de Crohn-like, descrevendo o quadro clínico, o processo de investigação diagnóstica, as abordagens terapêuticas e a evolução clínica do paciente. Realizar revisão bibliográfica de relatos de caso que abordem pacientes com a associação imunodeficiência comum variável e doença de Crohn-like. Enfatizar a importância do diagnóstico e tratamento precoces.

DESCRIÇÃO DO CASO: Um paciente masculino foi diagnosticado com imunodeficiência comum variável aos nove anos de idade e doença inflamatória intestinal inespecífica aos 10 anos, após realização de colonoscopia e biópsia colônica indicando moderado infiltrado linfoplasmocitário, eosinofílico e alguns neutrófilos em lâmina própria, com ausência de granulomas. Aos 14 anos, foi confirmado o diagnóstico de doença de Crohn-like por especialista após correlação entre história clínica e exames complementares. Atualmente com 18 anos, o paciente está em tratamento com imunoglobulina humana endovenosa, infliximabe e azatioprina, com estabilização do quadro clínico.

CONCLUSÕES: A revisão da literatura identificou 11 relatos de caso sobre a associação imunodeficiência comum variável e doença de Crohnlike, inexistindo trabalhos brasileiros, o que ressalta a raridade de tal associação. Neste relato, o paciente recebeu tratamentos amplos e empíricos devido à dificuldade em se chegar a um diagnóstico específico, o qual somente foi realizado aos 14 anos, quando se iniciou tratamento mais direcionado e individualizado. Apesar de atualmente estável, o paciente apresentou diversas complicações durante a investigação diagnóstica, o que ressalta a importância de diagnóstico precoce e tratamento mais preciso e dirigido às necessidades de saúde desses pacientes.

DESCRITORES: imunodeficiência de variável comum; doença de Crohn; imunidade; doenças do sistema imune; doença inflamatória intestinal.

\section{ABSTRACT}

AIMS: To report the case of a patient diagnosed with common variable immunodeficiency and Crohn-like disease, describing the clinical picture, the diagnostic investigation process, the therapeutic approaches and the clinical outcome of the patient. To carry out a literature review of case reports addressing patients with the common variable immunodeficiency and Crohn-like disease association. Emphasize the importance of early diagnosis and treatment.

CASE DESCRIPTION: A male patient was diagnosed with common variable immunodeficiency at nine years of age and with non-specific inflammatory bowel disease at 10 years, after colonoscopy and colonic biopsy indicated moderate lymphoplasmacytic, eosinophilic infiltrate and some neutrophils in lamina propria, with absence of granulomas. At age 14, the diagnosis of Crohn-like disease was confirmed by specialist after correlation between clinical history and complementary exams. Currently with 18 years of age, the patient is under treatment with intravenous human immunoglobulin, infliximab and azathioprine, with stabilization of the clinical picture.

CONCLUSIONS: The literature review identified 11 case reports on the association between common variable immunodeficiency and Crohn-like disease, with no Brazilian studies, which highlights the rarity of such association. In this report, the patient received extensive and empirical treatments due to the difficulty in reaching a specific diagnosis, which was only performed at age 14, when more targeted and individualized treatment was started. Although currently stable, the patient presented several complications during the diagnostic investigation, which emphasizes the importance of early diagnosis and more precise treatment, targeted to meet the health needs of these patients.

KEYWORDS: common variable immunodeficiency; Crohn disease; immunity; immune system diseases; inflammatory bowel disease. 
Abreviaturas: DC, doença de Crohn; DCL, doença de Crohnlike; DII, doença inflamatória intestinal; TNF, fator de necrose tumoral; ICV, imunodeficiência comum variável; IFN, interferon; Ig: imunoglobulina; IGEV, imunoglobulina humana endovenosa; IL, interleucina; $\mathrm{p}$, percentil; $\mathrm{PPD}$, purified protein derivative (teste tuberculínico).

\section{INTRODUÇÃO}

A imunodeficiência comum variável (ICV) é o segundo tipo de imunodeficiência primária mais comum, após a deficiência seletiva de imunoglobulina (Ig) A, com prevalência estimada entre 1:25.000 e 1:50.000 e incidência entre 1:50.000 e 1:200.000 [1-3]. Tem distribuição equivalente em ambos os sexos e seu início é mais comum entre os 20 e 40 anos de idade [4]. A doença é caracterizada por falha na diferenciação de linfócitos B em plasmócitos e linfócitos B de memória, gerando déficit na produção de imunoglobulinas séricas e, consequentemente, infecções recorrentes e persistentes, principalmente bacterianas e dos tratos respiratório e gastrointestinal. Tem sido relacionada a mutações autossômicas recessivas, como as dos genes CD278, TACI e CD19, porém essas mutações correspondem a somente $3 \%$ dos casos de ICV, sendo sua origem predominantemente idiopática [5].

A doença de Crohn (DC) é uma doença inflamatória intestinal crônica, transmural e idiopática, com incidência na América do Sul estimada entre 1-4:100.000, sendo mais frequente em áreas urbanas e no sexo feminino. O início da doença é mais comum entre os 15 e 30 anos de idade, com um segundo pico entre 60 e 80 anos [6]. A doença de Crohn-like (DCL) é uma doença inflamatória intestinal (DII) compatível com DC, porém decorrente de uma doença subjacente, como a ICV. O distúrbio imunológico causado pela ICV levaria à expressão de um processo inflamatório intestinal crônico semelhante à DC, a DCL [4]. Já a DC resulta da interação entre fatores genéticos, ambientais e imunológicos. Polimorfismos do gene NOD2 e a diminuição da diversidade da microbiota intestinal, principalmente da família Ruminococcaceae, parecem estar envolvidos na inflamação crônica da doença $[7,8]$.

É rara a associação entre ICV e DC; na literatura estima-se que $2-13 \%$ dos pacientes com ICV apresentam DII associada, sendo o diagnóstico de DC geralmente realizado anos após o diagnóstico de ICV [9]. A abordagem da associação dessas afecções também é justificada pelo fato de que o quadro clínico e os exames complementares de ICV e de DC apresentam semelhanças consideráveis entre si e com muitas doenças infecciosas e autoimunes, dificultando um diagnóstico preciso e postergando um tratamento específico.

Este relato de caso é bastante representativo do binômio imunodeficiência-autoimunidade e tem como objetivos relatar o caso de um paciente diagnosticado com imunodeficiência comum variável e doença de Crohn-like. O artigo descreve o quadro clínico, a investigação diagnóstica, as abordagens terapêuticas, a evolução clínica e as complicações decorrentes da associação entre essas doenças. Além disso o artigo inclui a revisão bibliográfica de relatos de casos de associação entre imunodeficiência comum variável e doença de Crohn-like e ressalta as dificuldades diagnósticas e a importância do diagnóstico e tratamento precoces.

\section{RELATO DO CASO}

Os dados que compõem esse relato de caso foram coletados através de prontuário manuscrito, após autorização do Comitê de Ética em Pesquisa da Faculdade de Medicina de Marília, sob o Parecer Consubstanciado de número 2.947.977. O paciente foi devidamente esclarecido sobre o relato e assinou o Termo de Consentimento Livre e Esclarecido.

O paciente, de gênero masculino, branco, com 18 anos por ocasião deste relato, nasceu a termo com peso adequado para a idade gestacional. Apresentou desenvolvimento neuropsicomotor adequado, porém teve dificuldade no ganho de peso e crescimento lento durante a infância. A mãe relata que aos quatro meses de idade o paciente começou a apresentar estomatite e episódios mensais de evacuações líquidas com fezes escurecidas, fétidas e sanguinolentas, distensão abdominal, irritabilidade e dor abdominal tipo cólica. A investigação da diarreia foi conduzida inicialmente com base em exames de fezes e hemograma, todos dentro dos parâmetros da normalidade.

A diarreia prosseguiu até os dois anos de idade, quando se iniciaram quadros repetitivos de febre alta, tosse produtiva, dor torácica e dispneia. As investigações apontavam pneumonias de repetição, com melhora após uso de antibióticos. Aos três anos de idade foram realizados exames para investigação das infecções de trato respiratório inferior recorrentes e da diarreia crônica. Os resultados da sorologia para HIV 1 e 2 e o rastreio para alergia alimentar foram negativos. A dosagem de imunoglobulinas séricas mostrou elevação de $\operatorname{IgE}(548.0 \mathrm{U} / \mathrm{mL})$, $\operatorname{IgA}(359.0 \mathrm{mg} / \mathrm{dL})$ e $\mathrm{IgG}(1112.0 \mathrm{mg} / \mathrm{dL})$. 
Aos quatro anos de idade, após inúmeras alterações em hemogramas de rotina anteriores, que indicavam anemia microcítica e hipocrômica, poiquilocitose, anisocitose, leptócitos e codócitos, foi solicitada a eletroforese de hemoglobinas, cujo resultado mostrou genótipo $\mathrm{HA}$, com $3 \%$ de hemoglobina $\mathrm{H}$, sugerindo traço alfa-talassêmico.

Aos sete anos, o paciente realizou lobectomia inferior de pulmão esquerdo, com posterior análise anatomopatológica, cuja microscopia indicou arquitetura geral preservada, brônquios e bronquíolos dilatados, preenchidos por secreção com infiltrado de neutrófilos, eosinófilos e algumas células gigantes multinucleadas, além de intenso infiltrado linfoplasmocitário ao redor dos bronquíolos, com formações de agregados linfoides e com centros germinativos proeminentes. As pesquisas para fungos e BAAR foram negativas. Foi feito o diagnóstico de bronquiolite folicular.

Aos nove anos o paciente foi internado por 15 dias, devido a broncopneumonia bilateral, tratada com ciprofloxacino. Após a internação foi encaminhado para o ambulatório de imunologia pediátrica da Faculdade de Medicina de Marília, onde iniciou investigação das infecções recorrentes de vias aéreas inferiores. Em consulta inicial foram observadas as seguintes alterações ao exame físico: lesões aftosas por toda a mucosa oral, língua geográfica, presença de tecido linfoide amigdaliano, hipertrofia de cornetos nasais $1+/ 4+$ e unhas em vidro de relógio; nenhuma alteração foi observada nos exames de tórax, coração e abdome.

Após anamnese e exame físico, as suspeitas foram de imunodeficiência primária, fibrose cística e tuberculose, sendo solicitados exames para determinação diagnóstica. Ao hemograma: hemoglobina $10,3 \mathrm{~g} / \mathrm{dL}$, hematócrito $33,5 \%$, moderada microcitose e hipocromia, leve policromasia, raros eliptócitos e esquisócitos, $2,7 \%$ de reticulócitos, 22.700 leucócitos $/ \mathrm{mm}^{3}$, $83 \%$ de segmentados, $13 \%$ de linfócitos típicos, com alguns neutrófilos com granulações tóxicas finas, e 546.000 plaquetas $/ \mathrm{mm}^{3}$. Proteína C reativa $1,84 \mathrm{mg} / \mathrm{L}$, ferritina 52,43 ng/mL. Exame parasitológico de fezes negativo, esteatócrito $0,5 \%$ e coprologia funcional sem alterações. Complementos séricos: C3 183,0 mg/dL e C4 36,9 mg/dL. Em exame de imunofenotipagem por citometria de fluxo (células $/ \mathrm{mm}^{3}$ ): linfócitos T CD3 1.234,14 (<percentil [p]10), linfócitos T CD4 191,04 $(<\mathrm{p} 10)$, linfócitos T CD8 578,56 (entre p10 e p50), linfócitos NK CD3-/CD16+/CD56+ 188,21 (entre p10 e p50), linfócitos B 871,0 (entre p50 e p90), linfócitos totais $2.105,32$, monócitos $1.305,24$ e granulócitos 25.710,60. A dosagem das imunoglobulinas séricas
(mg/dL) foram: IgA 24,8, IgM 17,6 e $\operatorname{IgG} 146,0$, todas abaixo de $\mathrm{p} 3$ para a idade. Subclasses $(\mathrm{mg} / \mathrm{dL})$ IgG1 86,0, IgG2 12,0, IgG3 10,0 e IgG4 1,0, as três primeiras abaixo de $\mathrm{p} 3$ e a última entre p3 e p10. Resultados das sorologias negativos para hepatites A, $\mathrm{B}$ e $\mathrm{C}$, mononucleose, rubéola, toxoplasmose, HIV e citomegalovírus. Dosagem de alfa-1-antitripsina $3,08 \mathrm{~g} / \mathrm{L}$, sódio no suor $24,4 \mathrm{mEq} / \mathrm{L}$, cloro no suor $27,2 \mathrm{mEq} / \mathrm{L}$, Teste de nitroblue tetrazolium $47 \%$ e prova tuberculínica (PPD) negativa. O paciente recebeu o diagnóstico de ICV após a análise dos exames, iniciando terapia com imunoglobulina humana endovenosa (IGEV) $15 \mathrm{~g}(400 \mathrm{mg} / \mathrm{kg})$ a cada 21 dias. A partir do diagnóstico, o paciente ficou aproximadamente um ano afastado das atividades escolares devido ao risco de contrair infecções oportunistas, prejudicando seu desenvolvimento escolar e sua interação social.

Com 10 anos, em consulta na gastroenterologia pediátrica para acompanhamento clínico, a mãe relatou diminuição da frequência da diarreia após o início do tratamento com IGEV, porém ainda apresentava inúmeras aftas em mucosa oral. Para esclarecimentos foram realizadas endoscopia digestiva alta $\mathrm{e}$ colonoscopia. A endoscopia não mostrou alterações em esôfago, piloro e duodeno, o estômago apresentava apenas enantema em antro e a pesquisa de Helicobacter pylori foi negativa. As biópsias indicaram fragmentos do estômago com gastrite crônica discreta; ausência de atividade neutrofílica, atrofia e metaplasia intestinal; fragmentos duodenais sem alterações; e fragmentos intestinais apresentando colite com moderada atividade neutrofílica e hiperplasia linfoide folicular. A colonoscopia, realizada até o ceco, indicou erosões e áreas de hiperemia por todo o cólon, com nodulações superficiais em cólon ascendente e ceco (Figura 1); sem biópsias. Após análise dos exames de imagem foi levantada a hipótese diagnóstica de DII inespecífica e receitado prednisona $40 \mathrm{mg} /$ dia, omeprazol $20 \mathrm{mg} /$ dia, espironolactona $25 \mathrm{mg} /$ dia, sulfametoxazoltrimetoprima $10 \mathrm{ml} /$ dia e sulfassalazina $500 \mathrm{mg}$, três vezes ao dia. Esta última foi trocada por azatioprina $50 \mathrm{mg} /$ dia após cinco meses, devido à não melhora das estomatites e dores abdominais.

Após nove meses de tratamento da diarreia foi realizada colonoscopia de rotina com pedido de biópsia, porém ocorreu perfuração de sigmoide durante o procedimento devido à intensa inflamação intestinal, sendo necessária internação hospitalar para tratamento de pneumoperitônio. A colonoscopia, com introdução do aparelho até $40 \mathrm{~cm}$ da borda anal, evidenciou presença de erosões por todo o cólon e distensibilidade preservada; biópsias colônicas 
indicaram moderado infiltrado linfoplasmocitário, eosinofílico e alguns neutrófilos em lâmina própria, com ausência de granulomas. O diagnóstico foi de colite inespecífica.
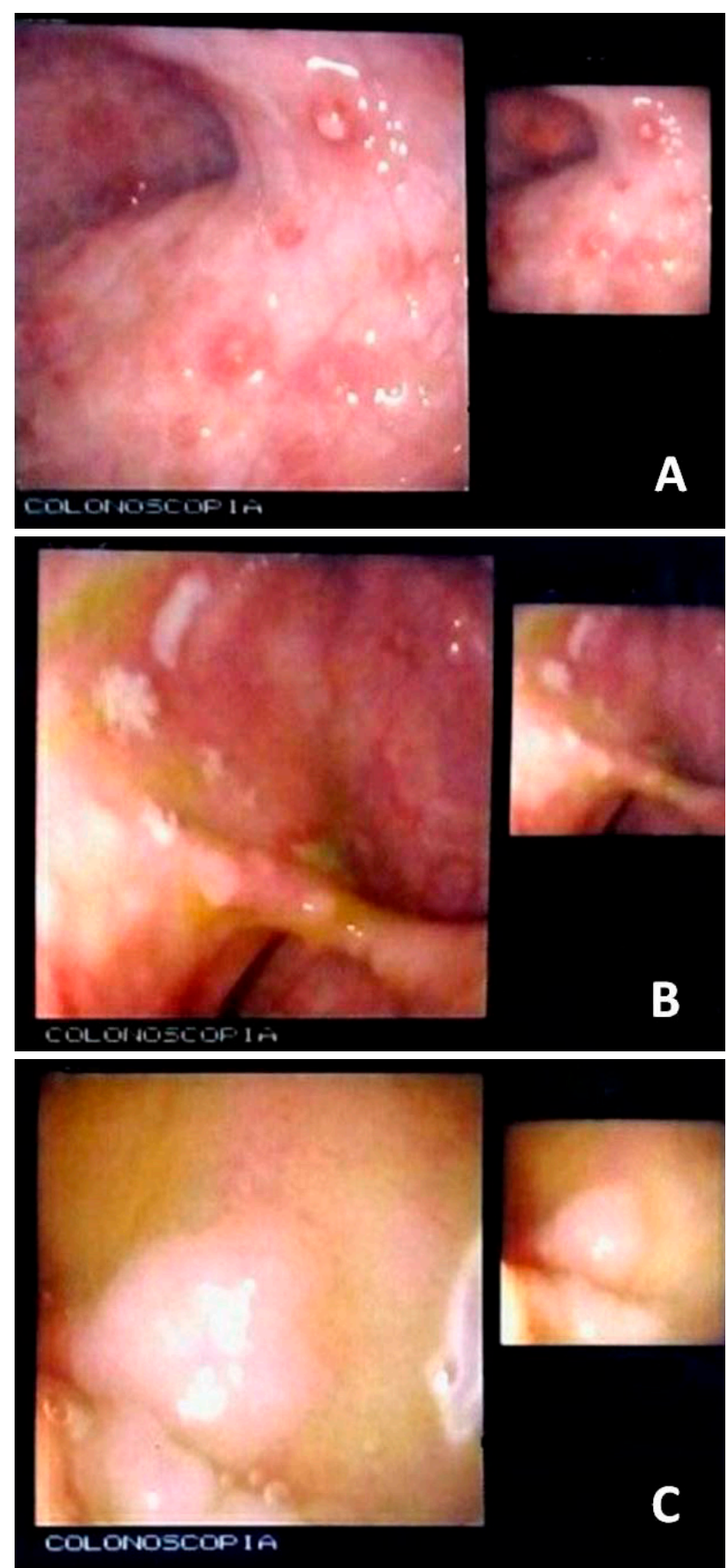

Figura 1. Colonoscopia realizada aos 10 anos, evidenciando áreas de lesões por todo o cólon e ceco. A. Numerosas ulcerações aftoides intercaladas com mucosa sã. B. Mucosa colônica apresentando intensa hiperemia e erosões difusas. C. Nodulações superficiais do tipo pedras de calçamento (cobblestone).
O acompanhamento nos ambulatórios de imunologia e gastroenterologia continuaram, com queixas esporádicas de dores abdominais e aumento da frequência dos episódios de diarreia, além de estomatite de repetição e frequentes infecções de vias áreas, porém sem necessidade de internação hospitalar ou uso de antibióticos endovenosos. Os níveis séricos de IgA, IgM e IgG continuaram abaixo de p3, mesmo com administração de IGEV a cada 21 dias.

Quando o paciente tinha 14 anos o caso foi discutido com um especialista que, ao correlacionar os dados clínicos e os exames complementares, chegou ao diagnóstico de DCL decorrente de ICV. Foi recomendado tratamento com IGEV $15 \mathrm{~g}$ a cada 21 dias, omeprazol $40 \mathrm{mg} / \mathrm{dia}$, prednisona $10 \mathrm{mg} / \mathrm{dia}$, infliximabe $5 \mathrm{mg} / \mathrm{kg}$ a cada 45 dias, metronidazol um comprimido diário nos primeiros 10 dias do mês e enzimas pancreáticas 25.000 UI cinco vezes ao dia, antes das refeições. Porém, três dias após a suspensão da azatioprina, o paciente apresentou dor abdominal e diarreia intensas, sendo reintroduzido esse medicamento.

Com 15 anos, após apresentar episódio de êmese intensa, diarreia e febre, o paciente foi submetido à endoscopia digestiva alta com biópsia, que indicou gastrite crônica discreta e esofagite crônica com atividade neutrofílica. Aos 16 anos o paciente iniciou acompanhamento no ambulatório de endocrinologia, sendo diagnosticado com osteopenia, atraso puberal e baixa estatura, manifestações que foram atribuídas à DCL e ao uso crônico de corticoide sistêmico. Iniciou então tratamento com testosterona intramuscular, vitamina $\mathrm{D}$ e carbonato de cálcio.

Atualmente, com 18 anos, o paciente vem mantendo um quadro clínico estável, fazendo uso de azatioprina $50 \mathrm{mg} / \mathrm{dia}$, infliximabe $10 \mathrm{mg} / \mathrm{kg}$ a cada 60 dias, IGEV $20 \mathrm{~g}(400 \mathrm{mg} / \mathrm{kg})$ a cada 30 dias, omeprazol $40 \mathrm{mg} /$ dia, carbonato de cálcio $1 \mathrm{~g} /$ dia, vitamina D $1.500 \mathrm{UI} /$ dia e ácido fólico $5 \mathrm{mg} / \mathrm{dia}$. Exames recentes mostraram hemoglobina $11,5 \mathrm{~g} / \mathrm{dL}$, hematócrito $36,2 \%$, moderada microcitose e hipocromia, leve policromasia e raros eliptócitos, 7.520 leucócitos $/ \mathrm{mm}^{3}, 54 \%$ de segmentados, $32 \%$ de linfócitos típicos, neutrófilos com ausência de alterações degenerativas e 264.000 plaquetas $/ \mathrm{mm}^{3}$; urinálise normal; ferritina $14,88 \mathrm{ng} / \mathrm{mL}$; ferro sérico $23 \mathrm{mcg} / \mathrm{dL}$; complemento C3 $193 \mathrm{mg} / \mathrm{dL}$ e C4 $36 \mathrm{mg} / \mathrm{dL}$; imunoglobulinas séricas com IgA $<5 \mathrm{mg} / \mathrm{dL} ; \operatorname{IgM}<5 \mathrm{mg} / \mathrm{dL}$ e IgG $468 \mathrm{mg} / \mathrm{dL}$; e ultrassonografia de abdome superior dentro dos padrões de normalidade. 


\section{DISCUSSÃO}

Esse caso de ICV associada à DCL representa uma associação nosológica rara, ainda não descrita na literatura brasileira e encontrada em somente 11 casos publicados na literatura médica pesquisada, sendo, entretanto, uma associação bastante representativa do binômio imunodeficiência-autoimunidade. O presente caso ratifica a maior prevalência dessa associação no sexo masculino, sendo que o diagnóstico de ICV precedendo o de DCL também é um dado encontrado nos casos relatados.

A revisão da literatura foi realizada na base de dados PubMed com o descritor "(common variable immunodeficiency) AND (Crohn disease OR colitis, ulcerative OR inflammatory bowel diseases)" e no Portal Regional da Biblioteca Virtual em Saúde com o descritor "(imunodeficiencia variável comum OR hipogamaglobulinemia) AND (doença Crohn OR colite ulcerativa OR doenças inflamatórias intestinais)". Não houve restrição de ano de publicação dos artigos e foram selecionadas publicações nos idiomas Inglês, Português e Espanhol. Foram encontrados 69 artigos, dos quais 11 eram relatos de caso da associação ICV e DC, porém um artigo deixou o diagnóstico interrogado entre DC e colite ulcerativa. Três artigos eram da Espanha, três dos Estados Unidos da América, um da Itália, um da Bélgica, um da Alemanha, um da Turquia e um do Japão. Não foi encontrado nenhum artigo em Português.

O intestino, maior órgão linfoide do organismo, contém linfócitos, que participam da imunidade local mediada por células, e produz imunoglobulinas. A inflamação crônica da mucosa intestinal presente na ICV e na DC pode ser explicada pelos desequilíbrios no processo de tolerância gastrointestinal e no controle da imunidade ativa decorrentes de alterações na produção de citocinas. Na ICV ocorre diminuição de IgA na mucosa intestinal, favorecendo o supercrescimento bacteriano, além de diminuição na produção de interleucina (IL)-2, IL-10 e fator de necrose tumoral (TNF)-alfa, e aumento de IL-12 e interferon (IFN)gama pelas células da lâmina própria. Já na $\mathrm{DC}$, ocorre produção excessiva de TNF-alfa, IL-23 e IL-17 [1-4, 10].

De acordo com a literatura, 2 a $13 \%$ dos pacientes com ICV apresentam DII associada, tendo como principais manifestações clínicas diarreia crônica, má absorção intestinal e perda de peso, sintomas apresentados pelo paciente deste relato. Além disso, o diagnóstico de DC geralmente é realizado anos após o diagnóstico de ICV, o que foi observado neste caso e em oito dos 11 artigos revisados [9]. Entre os 11 pacientes da literatura que apresentavam ICV e DC, 10 eram igualmente do sexo masculino, sendo necessários estudos adicionais que busquem a justificativa para essa distribuição epidemiológica.

$\mathrm{O}$ atraso em se diagnosticar ICV decorre em parte da sua mimetização endoscópica por outras doenças, como a doença celíaca e a DII. Já a demora no diagnóstico de DCL em crianças com imunodeficiência primária subjacente ocorre porque o exame histopatológico não consegue diferenciar colite ulcerativa de DC nessa faixa etária, descrevendo a inflamação intestinal como DII inespecífica, o que foi observado no presente caso. Além disso, a ICV e a DC possuem sinais e sintomas semelhantes, como diarreia crônica, estomatite, hiperplasia nodular linfoide e atrofia de vilosidades intestinais, o que dificulta o diagnóstico da associação de ambas as doenças [2].

Para a suspeita de ICV deve-se levar em consideração a história pregressa de infecções respiratórias e gastrointestinais de repetição, além de ser necessária a presença de todos os seguintes critérios, de acordo com as Sociedades Americana e Europeia de Imunodeficiência (critérios de 1999): diminuição de $\mathrm{IgG}$ sérica de pelo menos dois desvios-padrão abaixo do esperado para a idade e diminuição de pelo menos um dos isotipos IgM e IgA séricos; início da imunodeficiência após os dois anos de idade; ausência de iso-hemaglutinina e/ou pobre resposta às vacinas; e exclusão de outras causas de hipogamaglobulinemia [6].

No caso apresentado, três dos quatro critérios foram preenchidos, pois o diagnóstico de ICV foi realizado aos nove anos de idade; a dosagem das imunoglobulinas séricas $\operatorname{IgG}, \operatorname{IgM}$ e $\operatorname{IgA}$ estavam abaixo de p3 para a idade e as subclasses de $\operatorname{IgG}$ estavam abaixo dos valores de referência; além de terem sido excluídas outras causas de imunodeficiência primária e descartadas doenças infecciosas através de testes sorológicos e PPD negativos.

Além disso, na ICV a contagem sérica de linfócitos B geralmente é normal ou levemente reduzida, podendo existir alteração de linfócitos $\mathrm{T}$, como a diminuição da relação linfócitos T CD4/CD8. Neste caso ambas as características foram encontradas com base na imunofenotipagem, que indicou diminuição de linfócitos T CD3 e CD4 e valores normais de linfócitos T CD8, linfócitos B e linfócitos NK [6].

Já a DC é diagnosticada por meio de história e exame físico compatíveis, com confirmação radiológica, endoscópica e histológica e exclusão de agentes infecciosos causadores de inflamação [11]. A 
diarreia crônica é o sintoma mais comum, com fezes contendo muco e/ou sangue, mas também ocorrem dor abdominal, perda de peso, esteatorreia e estomatite. Os exames laboratoriais mostram aumento de proteína $\mathrm{C}$ reativa e de VHS, além de leucocitose. A endoscopia e a colonoscopia mostram inflamação de qualquer porção do trato gastrointestinal com áreas de lesão de mucosa interpostas por mucosa sã, ulcerações aftoides, úlceras longitudinais em formato de pedras de calçamento (cobblestone) em íleo e cólon, granulomas não caseosos, inflamação transmural e fibrose [6]. Neste relato, o diagnóstico de DCL só foi realizado por especialista em DII após associação da história de diarreia crônica e estomatites de repetição, sem melhora com uso de IGEV, diagnóstico prévio de ICV e colonoscopia indicando erosões e áreas de hiperemia por todo o cólon, com nodulações superficiais em cólon ascendente e ceco, além de biópsias intestinais mostrando colite com moderada atividade neutrofílica e hiperplasia linfoide folicular.

Em relação ao tratamento, os pacientes de todos os artigos revisados foram tratados para ICV com IGEV a cada 21 dias, sendo que somente um paciente não teve melhora do quadro clínico ou dos níveis séricos de IgG, necessitando mudar o tratamento para Ig subcutânea semanalmente, após isso com aumento progressivo de IgG sérica e diminuição dos sintomas gastrointestinais [12]. Muitos estudos mostraram que a IGEV não diminui a diarreia, a má absorção intestinal e os danos da mucosa em pacientes com ICV e DII; já a Ig subcutânea é absorvida lentamente, reduzindo a inflamação, além de possuir traços de IgA, diminuindo a suscetibilidade a infecções intestinais, o que não ocorre na IGEV [12]. Quanto ao tratamento da DC, somente dois dos 11 pacientes da literatura tiveram melhora dos sintomas gastrointestinais com uso isolado de corticoide sistêmico, sendo a prednisona o fármaco de escolha $[13,14]$. Um paciente faleceu antes do início do tratamento [15] e os outros oito pacientes só obtiveram melhora clínica após associações diversas de corticoide, aminossalicilatos (sulfassalazina ou mesalazina) [12], antineoplásico (6-mercaptopurina) [11], imunomoduladores (azatioprina) [16] e anti-TNF alfa (infliximabe ou adalimumabe) $[3,9,17]$, sendo que a associação de infliximabe e azatioprina foi benéfica em dois de oito casos, sem necessidade de uso concomitante de corticoide sistêmico $[4,18]$.

No presente caso, o paciente obteve melhora clínica considerável após administração de IGEV, com diminuição da diarreia e das infecções respiratórias, além de aumento significativo dos níveis séricos de
IgG. A DCL está sendo tratada com azatioprina e infliximabe, porém, foram realizadas diversas tentativas terapêuticas desde o diagnóstico de DII inespecífica aos 10 anos. $O$ paciente usou corticoide sistêmico em diversas dosagens, espironolactona, sulfametoxazoltrimetoprima, sulfassalazina, metronidazol e enzimas pancreáticas, todos sem sucesso. As dores abdominais e a diarreia intensificaram-se quando houve tentativa de suspender a azatioprina, mostrando a necessidade do seu uso contínuo para estabilização da DCL.

O tratamento para pacientes com associação de ICV e DCL é ainda experimental, com poucos estudos documentados sobre a eficácia e a tolerabilidade de cada plano terapêutico. A demora no diagnóstico da associação de ICV e DCL aliada a um plano terapêutico ainda não totalmente embasado prejudica a qualidade de vida desses pacientes, que sofrem internações hospitalares frequentes, têm queda na produtividade escolar e profissional, são submetidos a exames complementares excessivos e testes terapêuticos ineficazes, além de apresentarem maior risco de complicações, como fístulas, peritonite e abscessos na DCL e doenças granulomatosas na ICV $[4,6,8]$. O paciente estudado teve muitos desses comprometimentos, ressaltando a lobectomia inferior de pulmão esquerdo, perfuração de sigmoide durante colonoscopia investigativa, osteopenia, atraso puberal e baixa estatura decorrentes de DCL e uso crônico de corticoide sistêmico, além de prejuízo social e escolar devido às internações hospitalares frequentes.

Este caso demonstra a dificuldade diagnóstica da associação ICV-DCL, bem como suas complicações e prejuízos à qualidade de vida dos pacientes. A inexistência de um tratamento específico para a associação nosológica implica na necessidade de tentativas terapêuticas diversas, até que o paciente responda adequadamente a um tratamento individualizado. A comunidade médica precisa estar atenta aos sintomas inespecíficos, porém típicos do binômio imunodeficiência-autoimunidade, principalmente na infância, assim como correlacionar sempre os achados clínicos com os exames complementares, para agilizar o diagnóstico e minimizar as complicações. Além disso, pesquisas sobre a eficácia e a tolerabilidade dos tratamentos disponíveis para ICV-DCL devem ser estimuladas, para que se obtenha uma abordagem medicamentosa mais específica e eficiente.

\section{NOTAS}

Apoio financeiro

Este estudo não recebeu apoio financeiro de fontes externas. 


\section{Declaração de conflito de interesses}

Os autores declaram não haver conflitos de interesses relevantes ao conteúdo deste estudo.

\section{Contribuições dos autores}

Ambos os autores fizeram contribuições substanciais para concepção, ou delineamento, ou aquisição, ou análise ou interpretação de dados; e redação do trabalho ou revisão crítica; e aprovação final da versão para publicação.

Disponibilidade dos dados e responsabilidade pelos resultados

Os autores declaram ter tido total acesso aos dados obtidos e assumem completa responsabilidade pela integridade destes resultados.

\section{REFERÊNCIAS}

1. Daniels JA, Lederman HM, Maitra A, Montgomery EA. Gastrointestinal tract pathology in patients with common variable immunodeficiency (CVID): a clinicopathologic study and review. Am J Surg Pathol. 2007;31(12):1800-12. https://doi. org/10.1097/PAS.0b013e3180cab60c

2. Tegtmeyer D, Seidl M, Gerner P, Baumann U, Klemann C. Inflammatory bowel disease caused by primary immunodeficiencies - clinical presentations, review of literature, and proposal of a rational diagnostic algorithm. Pediatr Allergy Immunol. 2017;28(5):412-29. https://doi.org/10.1111/pai.12734

3. Akazawa Y, Takeshima F, Yajima H, Imanishi D, Kanda T, Matsushima K, Minami H, Yamaguchi N, Ohnita K, Isomoto H, Hayashi T, Nakashima M, Nakao K. Infliximab therapy for Crohn's-like disease in common variable immunodeficiency complicated by massive intestinal hemorrhage: a case report. BMC Res Notes. 2014;7:382. https://doi.org/10.1186/17560500-7-382

4. Vázquez-Morón JM, Pallarés-Manrique H, Martín-Suárez IJ, Benítez-Rodríguez B, Ramos-Lora M. Crohn's-like disease in a patient with common variable immunodeficiency treated with azathioprine and adalimumab. Rev Esp Enferm Dig. 2013;105(5):299-302. https://doi.org/10.4321/S1130-01082013000500010

5. Salzer U, Warnatz K, Peter HH. Common variable immunodeficiency - an update. Arthritis Res Ther. 2012;14(5):223. https://doi.org/10.1186/ar4032

6. Longo DL, Fauci AS, Kasper DL, Hauser SL, Jameson JL, Loscalzo J. Medicina interna de Harrison. $18^{\mathrm{a}}$ ed. Porto Alegre: AMGH Editora Ltda; 2013. p. 2477-95.

7. Greer RL, Morgun A, Shulzhenko N. Bridging immunity and lipid metabolism by gut microbiota. J Allergy Clin Immunol. 2013;132(2):25362. https://doi.org/10.1016/j.jaci.2013.06.025

8. Packwood K, Drewe E, Staples E, Webster D, Witte T, Litzman J, Egner W, Sargur R, Sewell W, Lopez-Granados E, Seneviratne SL, Powell RJ, Ferry BL, Chapel HM. NOD2 polymorphisms in clinical phenotypes of common variable immunodeficiency disorders. Clin Exp Immunol. 2010;161(3):536-41. https://doi.org/10.1111/j.1365-2249.2010.04216.x

9. Salda-a DC, Rubio IS. Immunodeficiencies and autoimmune diseases: common variable immunodeficiency and Crohnlike. Rev Esp Enferm Dig. 2016;108(8):520-3. https://doi.org/10.17235/reed.2015.3872/2015

10. Mannon PJ, Fuss IJ, Dill S, Friend J, Groden C, Hornung R, Yang Z, Yi C, Quezado M, Brown M, Strober W. Excess IL-12 but not IL-23 accompanies the inflammatory bowel disease associated with common variable immunodeficiency. Gastroenterology. 2006;131(3):748-56. https://doi.org/10.1053/j.gastro.2006.06.022

11. Bosworth BP, Sanders A, Maltz C. Common variable immunodeficiency masquerading as Crohn's ileocolitis. Inflamm Bowel Dis. 2006;12(2):151-2. https://doi.org/10.1097/01.MIB.0000200344.61707.9c

12. Sanges M, Spadaro G, Miniero M, Mattera D, Sollazzo R, D’Armiento FP, De Palma GD, Pecoraro A, Borrelli F, Genovese A, D'Arienzo A. Efficacy of subcutaneous immunoglobulins in primary immunodeficiency with Crohn's-like phenotype: report of a case. Eur Rev Med Pharmacol Sci. 2015;19(14):2641-5.

13. Mudter J, Wirtz S, Weigmann B, Tiede I, Tubbe I, Kiesslich R, Galle PR, Lehr HA, Neurath MF. Crohn's-like colitis in a patient with immunodeficiency associated with a defect in expression of inducible costimulator. Dig Dis Sci. 2006;51(4):711-7. https://doi.org/10.1007/s10620-006-3196-x

14. Strauss RG, Ghishan F, Mitros F, Ebensberger JR, Kisker CT, Tannous R, Younoszai MK. Rectosigmoidal colitis in common variable immunodeficiency disease. Dig Dis Sci.1980;25(10):798-801. https://doi.org/10.1007/BF01345303

15. Ünal B, Başsorgun Cİ, Gönülcü SC, Uçar A, Çelik F, Elpek GÖ. Cytomegalovirus colitis with common variable immunodeficiency and Crohn's disease. Case Rep Med. 2015;2015:348204. https://doi.org/10.1155/2015/348204

16. Salado CT, Carnerero EL, Moreno AP, Ramírez MC, Miguez AA, Justiniano JH, Galán JM. Tratamiento con inmunosupresores en paciente con enfermedad de Crohn e inmunodeficiencia común variable. Gastroenterol Hepatol. 2015;38(9):544-5. https://doi.org/10.1016/j.gastrohep.2014.09.008

17. Tuna Y, Koçak E, Köklü S. Common variable immunodeficiency associated with Crohn's disease. Acta Clin Belg. 2011;66(1):75-6. https://doi.org/10.1179/ACB.66.1.2062524

18. Nos P, Bastida G, Beltran B, Aguas M, Ponce J. Crohn's disease in common variable immunodeficiency: treatment with antitumor necrosis factor alpha. Am J Gastroenterol. 2006;101(9):2165-6. https://doi.org/10.1111/j.15720241.2006 .00763 5.x $\in$ 